\title{
ErHu Concerto in Eighties
}

\author{
Jindi Zhang \\ Art College, Shandong University, Weihai, 264209, China
}

\section{Keyword: Erhu concerto, Art}

\begin{abstract}
Modern music in twenties century is directly impacted by impressionism style, thus Debussy is universally acknowledged as the initiative person of modern music in twenties century. The new conception absorbing oriental elements is soon accepted by China that everything is waiting to be taken up in eighties, the concept of "chromatics" has a strong response in music field, the concept of aesthetics of East and West brings collisions, creative concept receives abstersion, the new concept has a fresh inspiration on the combination of folk music instrument and the modeling of sound shape, which has a distinctive difference from the traditional folk instruments, showing the diversification of creation phenomenon in the creative field. Erhu concertos get a special popularity during this period, which receives the development with the style of "Venting and Eruption", showing the production with thousands of multicolored gorgeously. This article chooses some common tracks of playing and teaching as there are too many works during this period.
\end{abstract}

\section{The art characters of Erhu}

\section{Erhu playing skills and the expansion of the stereo}

In eighties, the emerging of various types and styles big stone works stimulate the Erhu playing technology moving forward again. Skill of quick bow is more difficult, the application of shock interval is very common, interval relations is not limited to the application of natural interval, variant interval、 arpeggio single and chromatic scale has been applied in many works. For example, The Great Wall Capriccio is the earliest to use the skill, and the skills are very common applied in later works.

With the application of "impressionism" style and twelve-tone serial in the works, various kinds of sequence scale is used in untitled musical works, which seems there are rules to follow, actually ever-changing sequence scale makes the sound of erhu generating another fantastic, vacant, ignorant sound effects.

Sequence of the sound column breaks original musical scale relations; the traditional melodic development is no longer in existence. The utilization of the range of erhu has been reached its limit. The freedom of rhythm and beat breaks the law of the original, using a lot of irregular rhythm and beat. Modulation is frequently used.

Various kinds of interval relations and rhythm's harmonious relations of arpeggio scale 、 chromatic scale、 changes in tone scale and sequence of the sound column are the new skills during this period.

Skill training of transplantation tracks is more difficult; Such as Vagrant, Variations on a theme by Carmen, whose hard skills has been the new target of pursuit for the player. The characteristics of the technique can be summarized as high, difficult and new, strange during this period.

\section{The mingle of western composing skills and Chinese aesthetic}

Most of the subjects of the works in early eighties adopt the well-known folk music material, way of creation is mainly via recomposing great music which has been around for years, and works usually adopts single movement of body structure, mostly with a narrative plot and the nature of the singing with sound chamber lines. The model of using familiar melody for creation, being repeated used will easily cause sameness and forming a line production, which will loss impetus of hearing because the lack of innovation. At the same time, as the original existing melody of the dialogue 
between ecru and band and sound longitudinal acoustic effects, which will result the lack of overall conception because the diversion of the conceive and design of the longitudinal acoustic.

In 1982,the appearance of erhu concerto The great wall capriccio makes people find everything fresh and new, the cordial and freshness, fresh and alive note which all revealing the spirit of the nation, bring the impact of the hearing to people and shaking people's hearts, generating a sense of national pride and inspiring strong national cohesion of the people.

\section{Artistic and literary characteristics of the form and content of work}

1). Based on the tradition, seeking new thoughts

2). Breaking tradition, pursuit of exoticism and fanaticism

\section{Creation of erhu concerto and development of the art of playing}

Viewing and emulating performance of the national folk music recital and the national children's folk music solo game in 1982; Erhu invitational game in Beijing in 1985; national folk music instrument competition of "Shan Cheng Bei" Cup and international competition of Chinese instruments of "ART Cup"IN 1989. All above national instrumental music events are showing the quick development of folk music during eighties, which are also showing a strong desire to music works of new national instrumental in music industry during this period, and the emerging of large amount of new works further pushes the development of the actuating performance art. Such as The great wall capriccio, The First Erhu Concerto, which has become the turning point on the moving forward of erhu art.

The great wall capriccio continues the style of the majestic momentum of Sanmen Gorge Rhapsody, and it increases tolerance and implication on cultural connotation, which is more like a demeanor and ore mature and rich on praxes of technique, utilizing unprecedented manifestation no matter on the vocabulary theme types or forms of literature and construction of works, making the application by integrating Chinese and western culture, greatly expand the art intrinsic expression of erhu.

The deep of The great wall capriccio conforms to the deep temperament of erhu, besides that, Stature and verve、 the rhyme of the euphemism 、 composed of capability of Min Huifang expresses the greatness 、 pageantry 、 forceful and the profound vision of the Great Wall and also showing the temperament of the Chinese nation fully and delightfully. We can use the four simplest words of Min Huifang which represent the the traditional aesthetics of China to describe the works- emotion, temperament, character and rhyme. Emotion, having a sense of propriety, singing wonderfully with a silver voice and deep feeling and revealing naturally. Temperament, means artistic conception accessible, pure temperament, followed by bowing heartful, hidden track showing shape, entelechy showing far away, and reaching the scenic spots of "lifelikeness". Character, which means the style of noble, and it is the "personality"of Music. Rhyme, means the lingering charm of Music and the nation, showing the charm of beauty of operatic tunes of @erhu by using traditional techniques. "Breath" should be heavy, stable, deep and long. Clapper talk skills should be integrated into the expressive force and bearing and verve of the Music. The music expresses the most excellent quality of erhu, and that's part of its charm.

Train of thought for composition of Liu Jinwen and his bold atmosphere of the work style gives successors a lot of enlightenments, which causes Great changes of erhu composition in the future, and he has become the precursor of large concerto. Different themes and genres Erhu concerto works spring up in late period.

The First Erhu Concerto changes the style of deep temperament of The great wall capriccio,which showing its temperament of fashion via luxuriant sound, and has a higher requirements on voice quality of Erhu. Rapid sound group 、 big jump interval, change panels and overtone which forms a lot of note piece.

The First Erhu Concerto was firstly performed in Hong Kong in 1988,Mr Min Huifang, Who was the famous Erhu performer won the the great praises .But it didn't draw attention of national people 
until the tracks showed up on the International instrumental music concerto competition in 1994.The work is injected with more modern music element and should be waiting for a proper opportunity and when the music attitude of people has a proper changing which means people will have a higher perceive level of modern music, in this situation, people can accept new concept which breaks through the tradition. At the same time, the performance technique is also more perfect, everything is ready so that the works can be born at the right moment.

One of the most important character of The First Erhu Concerto is that the application of modern glossary enhancing the development of new erhu skill ,which has become the new leap of playing erhu art and erhu technique. Breakthrough application on rhythm expands the tension of erhu rhythm expression, which has become another important factor of the erhu works development in future.

With the erhu music going international, the expression of erhu music should not only be towards domestic people but also towards various people all over the world. We should keep many emphasized aspects on the culture communion so as to meet the aesthetic demand of different culture. As we put special emphasis on some aspect we should also pay attention to follow the rule of Chinese cultural characteristics of erhu, as we are showing the Chinese culture bearded by erhu instrument to foreign friends not just demonstrating a Chinese instrument.

In eighties, by the rising status of erhu, more and more professional composers pay much more attention on erhu, a lot of big stone works of erhu and concerto are all coming from the composer. As the differentiation of composers on the educational orientation 、 structure of knowledge, views and values, the works also showing their diversified phenomenon. Erhu and band Four Dreams of He Xuntian, Kao-hu of Zhao Xiaosheng, erhu and dual national orchestra concerto $I$ adds a new color to erhu art and mining the erhu expressive force.

\section{Representative works and characters}

The largest contribution of Debussy is putting harmonic to the functional application of "color", the color of harmonic is the main expressive factor to mold interval image. The abundant modes color interval of China and linear performance of the melody which all need the function of harmonic "color" to enrich themselves. China is in the stage of thirst for new knowledge in eighties, when the Erhu music symphony tide is coming, "Color" harmony for sound writing of Chinese music is more like a duck to water which plays a very important enlightenment function and application support.

Liu Wenjing and Min Huifang first visited US in 1978, they saw a huge picture of Great wall, heroic and brightness, all the people got excited, strong sense of national pride made Liu Jinwen passionate, and then she negotiated with Min Huifang to express people's perceptions of the ancient Great Wall by using the Chinese traditional folk music form, singing the Song of the Chinese nation's long history and looking forward to the beautiful future. The sense of national pride is the creation motivation of The great wall capriccio, which is also the soul of this work, the great ideas encountered three-year elaboration to complete, and we can feel that the writer written Chinese history and culture heartily.

The great wall capriccio suits both refined and popular tastes and is the combination of Chinese and western. On the use of traditional culture, mainly depicting the acura history and with the guqin music elements, showing the demeanor temperament of "big elegance"; At the same time, drawing folk tone such as Beijing dialect, talking and singing to reflect the national customs of " folk music". "Elegance" and "popular" express the multiple Chinese cultures perfectly. On the application of Chinese and Western culture, they use big solo kanadeai theme matching with identity of "Great wall" to interpret Chinese culture via the unique cavity of erhu instrument performance.

Integrated with writing techniques such as the use of Chinese and western style, form, techniques to reach the state of harmony, with a high artistic taste and resonating and popular with the masses, which is very successful work. Works both in erhu art and erhu performance has a step over compared with erhu works in the early period. Broadening and deepening the expressive force of the erhu, exploiting new areas of Erhu, which has become another turning point in the development of erhu art. And playing a leading role for successor, raising great mass fervor of Erhu concerto composing. 
As Guan Naizhong has a special experience of Chinese and foreign life, which brings him the concept of advanced culture of the world trend and a keen sense of smell in the field of erhu creation, With this advantage, he combined his own experience and wisdom with the experience of predecessors' creation, he made a more progressive western creation concept to create a new concept of erhu music creation firstly. Like his own concept: What kind of idea will produce what kind of behavior

Mr. Guan graduated from the Central Music College in 1961 and has learnt to play the violin with his father when he is a child. With a solid foundation of western music and wealthy life experience, experienced the "516" counter-revolutionary organization cultural events once worked and lived in Hong Kong, Taiwan, Canada. He has created many realistic and national style works in his early writings, such as Travel in Lhasa, The first erhu concerto is his first erhu works in the field of erhu creation. The breakthrough of title music and the use of the rhythm, applying of the development of color and acoustics, and the creation of reflective thinking, the creation of the breakthrough broaden a new space of the erhu music creation, expanding the new expression of erhu art. He has explored a new path for the diversification of erhu music creation, and has become the leading figure in modern erhu music creation boom period,

He has formed a new transition branch of music creation of erhu concerto after "Great Wall scribblings", keeping pace with the Times and complying with the requirements of the times.

\section{Erhu concerto teaching and theoretical research}

The second session of the national erhu teaching was held in xi 'a college of music in 1980. The third national erhu art college teaching materials seminar was held in Tianjin music academy in 1988

The $80 \mathrm{~s}$ is the peak period of creation of erhu concerto, diverse work style prompted players to have diversified performance ability, the work performance of Erhu concerto has more and higher demand of erhu technology, which has surpassed technical difficulties of other erhu works, and it promotes the improving and enhancing of the erhu teaching and the erhu theory.

Because in violation of the principle of use for reference and into study, with the western literary thoughts and artistic creation technique was introduced into China, Chinese music aesthetic thinking is raising a transformation, the diversity of erhu music development becomes inevitable. At the same time, music education of the most high-end, the cutting edge of the central, Shanghai Music College is a banner leading other music education development in China, the flag directs the development of education.

In 1987, Mr. Wang Guotong concluded his instrumental performance and the practice of teaching in more than 20 years combined with the development of erhu art, actually the technical difficulties and breadth of erhu far surpass the content of the teaching material. Revising and making up 108 items of Scale Study of erhu, teaching material is combined of early, middle and senior stage step by step, including the traditional and western scale, sequence and the practice of arpeggio. pentatonic scale, heptachord, chromatic scale whole-tone scale, practicing of arpeggio, rhythm, martele, sound pattern sequence, alternate sequence, triad sequence, octave sequence. Absorbing the most forefront new technology of erhu art, such as full scale techniques and atonal technique. As Zhao Feng write in the Initial of the book: the book is the first practice materials of erhu scale, which will have profound impact of the popularization and improve the erhu instrument and teaching specialization of erhu.

\section{Conclusion}

Generally speaking, the overall development characters of eighties is that the music length is developing towards big stone works, using larger movement model on the musical structure, single movement and multiple movement are mixed used. The application of chromatic scale、chromatic interval、shock interval、 broken chord arpeggio、 irregular rhythm is the new language expression method during this period, which showing the character of grand music them v colors revealing 
momentum. The etude is developing more specialized training requirements according to the increasing difficulty of fast bow skill.

\section{References}

[1] Qiao Jianzhong, Song of praise of Love - Listen to the erhu concerto <Mother $>$ Impression, People's music, 1992(5), 22. 